\title{
BMJ Open Effect of a redesigned fracture management pathway and 'virtual' fracture clinic on ED performance
}

\author{
J Vardy, ${ }^{1}$ P J Jenkins, ${ }^{2} \mathrm{~K}$ Clark, ${ }^{1} \mathrm{M}$ Chekroud, ${ }^{1} \mathrm{~K}$ Begbie,,${ }^{1}$ I Anthony, ${ }^{2}$ \\ L A Rymaszewski, ${ }^{2}$ A J Ireland ${ }^{1}$
}

To cite: Vardy J, Jenkins PJ, Clark K, et al. Effect of a redesigned fracture management pathway and 'virtual' fracture clinic on ED performance. BMJ Open 2014;4:e005282.

doi:10.1136/bmjopen-2014005282

- Prepublication history for this paper is available online. To view these files please visit the journal online (http://dx.doi.org/10.1136/ bmjopen-2014-005282).

Received 17 March 2014 Revised 28 May 2014 Accepted 2 June 2014

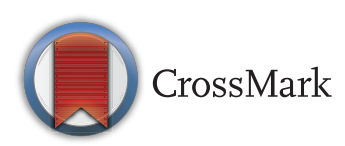

${ }^{1}$ Department of Emergency Medicine, Glasgow Royal Infirmary, Glasgow, UK ${ }^{2}$ Department of Orthopaedic Surgery, Glasgow Royal Infirmary, Glasgow, UK

\section{Correspondence to}

Dr A J Ireland;

alastair.ireland@ggc.scot.nhs. uk

\section{ABSTRACT}

Objectives: Collaboration between the orthopaedic and emergency medicine (ED) services has resulted in standardised treatment pathways, leaflet supported discharge and a virtual fracture clinic review. Patients with minor, stable fractures are discharged with no further follow-up arranged. We aimed to examine the time taken to assess and treat these patients in the ED along with the rate of unplanned reattendance.

Design: A retrospective study was undertaken that covered 1 year before the change and 1 year after. Prospectively collected administrative data from the electronic patient record system were analysed and compared before and after the change.

Setting: An ED and orthopaedic unit, serving a population of 300000 , in a publicly funded health system.

Participants: 2840 patients treated with referral to a traditional fracture clinic and 3374 patients managed according to the newly redesigned protocol.

Outcome measures: Time for assessment and treatment of patients with orthopaedic injuries not requiring immediate operative management, and 7-day unplanned reattendance.

Results: Where plaster backslabs were replaced with removable splints, the consultation time was reduced. There was no change in treatment time for other injuries treated by the new discharge protocol. There was no increase in unplanned ED attendance, related to the injury, within 7 days $(p=0.149)$. There was a decrease in patients reattending the ED due to a missed fracture clinic appointment.

Conclusions: This process did not require any new time resources from the ED staff. This process brought significant benefits to the ED as treatment pathways were agreed. The pathway reduced unnecessary reattendance of patients at face-to-face fracture clinics for a review of stable, self-limiting injuries.

\section{INTRODUCTION}

All patients with acute fractures have traditionally been referred to a fracture clinic soon after injury. When there is a need for an orthopaedic opinion in the emergency

\section{Strengths and limitations of this study}

- This study used prospectively collected administrative data for a large number of consecutive patients.

- It provides useful information to demonstrate that there was no significant increase in workload in the emergency department.

- The use of removable splints in preference to plaster backslabs resulted in reduced treatment time.

- This study cannot confirm a causal relationship between these findings, only an association.

- This study design cannot detect any longer term orthopaedic consequences of this new process such as missed diagnosis or late complication detection. Further studies should be performed to examine this.

department (ED), the patient is often initially reassessed by the most junior member of the orthopaedic team. Both these issues can lead to increased time in the ED, along with unnecessary subsequent clinic visits. A collaboration between Orthopaedic and Emergency Medicine Services at our institution has resulted in a redesigned fracture management system with agreed guidelines on treatment, direct admission criteria and leaflet supported discharge from the ED, along with implementation of a 'Virtual' fracture clinic (VFC) system for orthopaedic review. Although other institutions have introduced systems of outpatient trauma triage and nurse-led clinics, ${ }^{1}{ }^{2}$ there are no reports of units implementing protocols for the definitive management of many simple, stable fractures completely within the ED.

The redesigned process had clear benefits for the orthopaedic service ${ }^{3}$ and appeared more convenient to patients but ED staff were concerned that additional time and responsibility was being taken in the ED to carefully explain the follow-up arrangements and that this might have an adverse effect on 
patient flow. In addition, there was potential for patients to reattend the ED as a result of problems, whereas prior to redesign they would already have attended a traditional fracture clinic. This may be offset by the use of splints rather than backslabs, which may result in timesavings in application.

The aims of this study were to examine the time taken to manage common musculoskeletal injuries in the ED, before and after this process change, along with the seven-day reattendance rate.

\section{PATIENTS AND METHODS}

A redesigned pathway was introduced in our institution in October 2011. It consisted of 'Direct ED Discharge' for the most minor, stable fractures and a VFC review of all other injuries that did not require immediate admission. Isolated fifth metacarpal neck fractures, paediatric greenstick, clavicle and torus wrist fractures, mallet fingers, isolated radial head fractures, elbow 'fat pad positive' injuries and fifth metatarsal fractures were discharged by ED staff with appropriate treatment and written advice, but no routine follow-up. A telephone hotline to the fracture service was available for any subsequent questions. All other fractures that did not require immediate inpatient management had their clinical record and digital radiographic images reviewed at a thrice-weekly VFC by a consultant orthopaedic surgeon. There were three possible outcomes: virtual discharge, nurse-led clinic review or consultant specialty clinic review. The specialty clinics covered hand and wrist, foot and ankle, shoulder and elbow and knee. All patients were then telephoned and a management plan agreed. Use of removable wrist and ankle splints was encouraged in the ED instead of plaster application for the majority of outpatient-managed fractures involving the wrist, ankle and foot. More detailed protocols and advice leaflets are available online at http://www. fractureclinicredesign.org

During a one-year period from October 2011 to September 2012, there were 6385 patients treated using this new protocol. The ED direct discharge rate was $23 \%$ $(\mathrm{n}=2115)$ and $4270(67 \%)$ were discussed at the VFC. Following the VFC, 1687 patients were discharged with no further face-to-face review. The remainder were reviewed at a specialty trauma clinic or a nurse-led clinic. After the VFC discussion, patients were contacted by telephone, with a further letter sent to the patient and general practitioner. In this series of patients discharged from the VFC with no further face-to-face review $(\mathrm{n}=1687)$, telephone contact was made with 1208 $(72 \%)$ while messages were left on voicemail for 258 $(15 \%)$. No telephone contact was made with 221 patients (13\%). A written summary of the discussion was sent to all patients. In a further development to the process, a senior orthopaedic registrar (specialty trainee year 3 and above) was available for advice and it was agreed that all but the most complex patients could be directly admitted after discussion, rather than wait for a further duplication of review.

A retrospective study was carried to examine a period before and after the introduction of the new process. The study period was from October 2010 to November 2012. Ethical permission was not required as this was classed as a service audit and used pre-existing management level, anonymised data. The primary database used was EDIS (Emergency Department Information System, Omnis Software). Data were collected prospectively at the time of the clinical episode. A final discharge diagnosis and treatment outcome was recorded by the responsible clinician, along with the time of presentation and discharge. During this period, there were 167735 ED visits.

A control group of patients were identified. This group had sustained a simple ankle ligament sprain $(n=4060)$. This minor injury was completely managed by the ED throughout both study periods, without any change in management.

The study group was composed of a well-defined group of patients with orthopaedic injuries (isolated fracture of the fifth metacarpal, radial head, metatarsal and ankle (not requiring surgery) and radial styloid (not requiring surgery)). We chose these injuries as they were most reliable and numerous to identify within the data set. They were 'indicator' injuries that acted as markers for the effect of the process on similar groups (ie, those treated with splints vs plaster backslabs). It was not always possible restrospectively to identify whether a patient had been referred to the VFC; therefore, this group is analysed together. Therefore, within the second group were patients who were directly discharged with no follow-up, patients referred for discussion at the VFC (stable, undisplaced ankle fractures) and patients with fractures that were managed with removable splints in preference to plaster backslabs after the process change. The treatment time was calculated from the ED database as the time from initial clinician contact to discharge. This was chosen as it represented the time taken to assess and manage these injuries and was independent of waiting time. To examine the effect on unplanned returns, the data set was queried to identify patients with a subsequent episode related to the original attendance within 7 days. The discharge diagnosis was examined to determine the reason for return. There were 2840 patients who met the inclusion criteria before the change, and 3374 patients after.

\section{Statistical analysis}

To compare the ED treatment time for patients who were discharged and admitted, independent sample Student $t$ tests were used. The mean difference, 95\% CI and $\mathrm{p}$ values were reported. Two-tailed tests were used and the level of significance was set at $\mathrm{p}<0.05$. Where categorical data were compared, a $\chi^{2}$ test (Fisher's exact test if any cell had less than five observations) was used and reported with an OR, 95\% CI and $\mathrm{p}$ value. The 
Table 1 Time from attendance to discharge (minutes) for (1) an injury completely treated by the ED and (2) injuries eligible for the new process

\begin{tabular}{|c|c|c|c|c|}
\hline & \multicolumn{4}{|c|}{ Treatment time (mean, min) } \\
\hline & Before & After & Mean difference (min, 95\% Cl) & p Value \\
\hline \multicolumn{5}{|l|}{ (1) ED managed injury $(n=4060)$} \\
\hline Ankle sprain $(n=4060)$ & 45.0 & 45.1 & $0.1(-1.5$ to 1.8$)$ & 0.888 \\
\hline \multicolumn{5}{|c|}{ (2) Managed by new process (combined ED discharge and VFC referral) $(n=2154)$} \\
\hline 5th Metacarpal $(n=566)$ & 51.6 & 54.3 & $2.7(-2.6$ to 8.1$)$ & 0.316 \\
\hline Radial head $(n=273)$ & 52.2 & 52.4 & $0.2(-6.0$ to 6.5$)$ & 0.948 \\
\hline Metatarsal $(n=687)$ & 117 & 119 & $2.0(-5.1$ to 9.1$)$ & 0.571 \\
\hline Ankle fracture $(n=539)^{*} \dagger$ & 71.3 & 65.2 & $-6.1(-12.1$ to -0.1$)$ & 0.046 \\
\hline Radial styloid $(n=89)^{*}$ & 62.8 & 49.6 & $-13.2(-25.3$ to -1.2$)$ & 0.032 \\
\hline
\end{tabular}

admission time was compared using an analysis of variance test. Post hoc comparison with a Tukey correction for multiple testing was made of each step in the process, and a multiplicity adjusted $\mathrm{p}$ value was reported.

\section{RESULTS}

There was no change in the treatment time for patients with ankle sprains. This group was completely managed by the ED and their treatment was unchanged during the study ( $p=0.888$; table 1$)$. They therefore acted as a control group. Similarly, for patients eligible for the redesigned process, there were no increases in treatment time for fifth metacarpal fractures $(p=0.316)$, radial head fractures $(p=0.948)$ and fifth metatarsal fractures $(p=0.571)$. For injuries that were treated with removable splints, there was a reduction in treatment times for radial styloid fractures $(\mathrm{p}=0.046)$ and for simple, stable ankle fractures $(\mathrm{p}=0.032)$; (table 1$)$.

There was no change in the overall unplanned reattendance rate before or after the change $(\mathrm{p}=0.149)$ (table 2). There was, however, a decrease in reattendance in the ED in the cases of patients who had failed to attend a planned fracture clinic appointment ( $\mathrm{p}=0.044$; table 2).

\section{DISCUSSION}

This study shows that ED performance was not adversely affected by the introduction of a virtual fracture review process. The time to discharge was not increased as a result of having to provide definitive explanation and advice. The time in the department was reduced in the case of radial styloid and non-operative ankle fractures. This may have been due to the time saving created by the use of removable splints in preference to plaster backslabs. Removable splints have previously been described in the successful early treatment of these fractures. ${ }^{4}$

Many stable fractures will heal without any further intervention. ${ }^{5-16}$ Many of these patients do not require further review, but they do require a careful explanation of their condition, treatment plan, likely course and prognosis. For discharged patients, this is an ED clinician backed up by agreed written information. For patients reviewed in the VFC, this is further emphasised by telephone communication with the patient and review arranged - at the most appropriate clinic-where necessary. Such processes have benefits for patients by reducing unnecessary attendance and interventions such as repeat radiographs, ${ }^{16-18}$ along with improved orthopaedic training experiences. ${ }^{19}$

As a profession, we should move towards patientcentred care, a key point of which is to enable patients to participate in planning follow-up. Review is only necessary for many stable injuries where a problem has developed and recovery does not meet expectations. Patients can be involved in decisions about follow-up based on individual factors, not least of which is convenience for them rather than the reviewing team, provided they are supported by clear fracture management advice provided initially by their ED consultation and reinforced by discharge information leaflets or further telephone consultation and have the option to seek further

Table 2 Seven day unplanned re-attendance related to initial injury

\begin{tabular}{|c|c|c|c|}
\hline & Before (n) & After (n) & p Value \\
\hline $\begin{array}{l}\text { Total ED } \\
\text { attendances }\end{array}$ & 2840 & 3374 & \\
\hline $\begin{array}{l}\text { Increasing pain or } \\
\text { concern }\end{array}$ & 32 & 46 & 0.408 \\
\hline $\begin{array}{l}\text { Plaster/splint/sling } \\
\text { problem }\end{array}$ & 27 & 18 & 0.056 \\
\hline $\begin{array}{l}\text { Missed clinic } \\
\text { appointment }\end{array}$ & 4 & 0 & 0.044 \\
\hline $\begin{array}{l}\text { Unable to manage } \\
\text { at home }\end{array}$ & 2 & 1 & 0.531 \\
\hline Wound problem & 1 & 0 & 0.457 \\
\hline Total & $70(2.5 \%)$ & $65(1.9 \%)$ & 0.149 \\
\hline
\end{tabular}


advice if needed. This paper has focused on the effects of the process on the ED. In separate work, we have examined patient satisfaction with the process. In a group of patients with suspected and definite radial head fractures, $90 \%$ were managed with direct discharge from the ED. ${ }^{3}$ The satisfaction rate ranged from $87 \%$ to $95 \%$. $^{3}$ Furthermore, in this group, there were only two patients who underwent late surgery for a late complication. Both of these patients recontacted the fracture clinic when their pain and restriction of movement did not settle in the time frame that had been discussed with them during their initial ED visit. These patients would not have undergone surgery at an earlier point as it is a general principle to treat all these injuries with observation in the first instance, and to only intervene in the case of late complications.

Agreed guidelines (between the ED and orthopaedic departments) are vital to the success of a redesigned programme. Such consensus between two teams reassures ED clinicians that their decisions are appropriate and supported by departmental guidance, ensures a consistent approach and reduces the requirement to seek advice from ED seniors or orthopaedic staff. Some advances in technology have facilitated this redesign, which would not previously have been possible. Digital imaging and the ability to view images in multiple locations were essential for decision making and vital to the success and quality control of this process. The almost universal use of mobile phones has enabled more reliable communication with patients while the reduced cost of plastic/velcro splints has made the use of plaster -and subsequent follow-up-less necessary.

One of the strengths of this study was the use of data that were prospectively collected for administrative reasons. It describes the pragmatic, real-life effect of introducing a new process. It was possible to examine the process and outcomes of a large number of patients over the time period. We were able to make a comparison with a control group of patients with injuries that had always been treated by the ED without referral (ankle sprains). This reduced the effect of any other simultaneous variation on the process that was not measured. The weakness of this study was the inability to draw a causal relationship between the process change and any observed differences in consultation times, reattendance rates or time to admission. We were also not able to exclude the effects of other changes in the ED that may have impacted on 'breach' proportions. Owing to the variation in admission processes between specialties, it was not possible to select an appropriate control group. It was also a retrospective study that was potentially subject to bias. The retrospective nature also resulted in our being unable to determine the ultimate destination of several patients. We therefore chose to analyse the patients managed under the new protocol together as one group. There was also the possibility of making a type II error, particularly in the analysis of reattendance rates. We mitigated this by including as large a patient group as possible and reducing the number of categories and subgroups examined by us. This study did not examine the short-term and long-term clinical outcomes of these patients. We have already published the functional outcome and satisfaction for patients with radial head fractures treated with this protocol. ${ }^{3}$ We are currently undertaking a wider study of these outcomes. Future studies should examine patient satisfaction with this process, along with the long-term functional outcomes of a prospective series of patients treated using this protocol.

From the ED perspective, this redesign process has improved ED performance by reducing the time spent in the department for some patients with fractures without leading to an increase in 'unplanned re-attendances'. It has also demonstrated that a collaborative approach can lead to more efficient use of resources whilst allowing people with fractures to be more involved in the planning care tailored to their own needs. We have provided further information about our redesign online at http://www.fractureclinicredesign.org.

Contributors JV planned the study, collected and analysed the data and cowrote the manuscript. PJ analysed the data and cowrote the manuscript. $M C$ and $\mathrm{KC}$ collected and analysed the data. KB managed the databases used and constructed the queries and also participated in the analysis. IA planned the study and cowrote the manuscript. LR and Al planned the study, cowrote the manuscript and act as coguarantors for the study.

Funding This research received no specific grant from any funding agency in the public, commercial or not-for-profit sectors.

Competing interests $L R$ is in receipt of an unrestricted grant from the Scottish Government Health Department Whole System Patient Flow programme.

Provenance and peer review Not commissioned; externally peer reviewed.

Data sharing statement No additional data are available.

Open Access This is an Open Access article distributed in accordance with the Creative Commons Attribution Non Commercial (CC BY-NC 4.0) license, which permits others to distribute, remix, adapt, build upon this work noncommercially, and license their derivative works on different terms, provided the original work is properly cited and the use is non-commercial. See: http:// creativecommons.org/licenses/by-nc/4.0/

\section{REFERENCES}

1. Beiri A, Alani A, Ibrahim T, et al. Trauma rapid review process: efficient out-patient fracture management. Ann $R$ Coll Surg Engl 2006;88:408-11.

2. Williams SC, Hollins D, Barden-Marshall S, et al. Improving the quality of patient care: patient satisfaction with a nurse-led fracture clinic service. Ann R Coll Surg Engl 2003;85:115-16.

3. Jayram PR, Bahattacharyya R, Jenkins PJ, et al. Patient satisfaction following management of radial head and neck fractures in a virtual fracture clinic. J Shoulder Elbow Surg 2014;23:297-301.

4. Williams KG, Smith G, Luhmann SJ, et al. A randomized controlled trial of cast versus splint for distal radial buckle fracture: an evaluation of satisfaction, convenience, and preference. Pediatr Emerg Care 2013;29:555-9.

5. Ahn H, Court-Brown CM, McQueen MM, et al. The use of hospital registries in orthopaedic surgery. J Bone Joint Surg Am 2009;91 (Suppl 3):68-72.

6. Bansal R, Craigen MA. Fifth metacarpal neck fractures: is follow-up required? J Hand Surg Eur Vol 2007;32:69-73.

7. Cakir H, Van Vliet-Koppert ST, Van Lieshout EM, et al. Demographics and outcome of metatarsal fractures. Arch Orthop Trauma Surg 2011;131:241-5. 
8. Calder JD, Solan M, Gidwani S, et al. Management of paediatric clavicle fractures-is follow-up necessary? An audit of 346 cases. Ann R Coll Surg Engl 2002;84:331-3.

9. Duckworth $A D, M c Q u e e n$ MM, Ring D. Fractures of the radial head. Bone Joint J 2013;95-b:151-9.

10. Duckworth AD, Watson BS, Will EM, et al. Radial head and neck fractures: functional results and predictors of outcome. $J$ Trauma 2011;71:643-8.

11. Herbertsson $\mathrm{P}$, Josefsson $\mathrm{PO}$, Hasserius $\mathrm{R}$, et al. Uncomplicated Mason type-II and III fractures of the radial head and neck in adults. A long-term follow-up study. J Bone Joint Surg Am 2004;86-A:569-74.

12. Herbertsson $P$, Josefsson $P O$, Hasserius R, et al. Displaced Mason type I fractures of the radial head and neck in adults: a fifteen- to thirty-three-year follow-up study. J Shoulder Elbow Surg 2005;14:73-7.

13. Malmvik J, Herbertsson $P$, Josefsson PO, et al. Fracture of the radial head and neck of Mason types II and III during growth: a 14-25 year follow-up. J Pediatr Orthop B 2003;12:63-8.
14. Martin AG. Weber B ankle fracture: an unnecessary fracture clinic burden. Injury 2004;35:805-8.

15. Symons S, Rowsell M, Bhowal B, et al. Hospital versus home management of children with buckle fractures of the distal radius. A prospective, randomised trial. J Bone Joint Surg Br 2001; 83:556-60.

16. van Bosse $\mathrm{HJ}$, Patel RJ, Thacker M, et al. Minimalistic approach to treating wrist torus fractures. J Pediatr Orthop 2005;25:495-500.

17. Chaudhry S, DelSole EM, Egol KA. Post-splinting radiographs of minimally displaced fractures: good medicine or medicolegal protection? J Bone Joint Surg Am 2012;94:e128.

18. Ghattas TN, Dart BR, Pollock AG, et al. Effect of initial postoperative visit radiographs on treatment plans. J Bone Joint Surg Am 2013;95: e571-4. S571.

19. Murray O, Christen K, Marsh A, et al. Fracture clinic redesign: improving standards in patient care and interprofessional education. Swiss Med Wkly 2012;142:w13630. 


\section{Correction}

Vardy J, Jenkins PJ, Clark K, et al. Effect of a redesigned fracture management pathway and 'virtual' fracture clinic on ED performance. BMJ Open 2014;4:e005282.

The funding and competing interests statement in this paper are incorrect. The correct statements are:

Funding This study was funded by the Quality and Efficiency Support Team of the Scottish Government Health Department through the Whole System Patient Flow workstream.

Competing interests None.

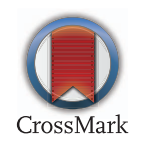

BMJ Open 2014;4:e005282. doi:10.1136/bmjopen-2014-005282corr1 